\title{
Performance Enhancement of QPSK Modulation Using Hybrid QPSK-modified MPPM in Optical Fiber Communications
}

\author{
Hossam Selmy $^{(1)}$, Hossam M. H. Shalaby ${ }^{(2)}$, Zen Kawasaki ${ }^{(3)}$ \\ ${ }^{(1)}$ Egypt-Japan University of Science and Technology (E-JUST), Alexandria, Egypt, hossamselmy@yahoo.com \\ ${ }^{(2)}$ Egypt-Japan University of Science and Technology (E-JUST), Alexandria, Egypt, shalaby@ieee.org \\ ${ }^{(3)}$ Egypt-Japan University of Science and Technology (E-JUST), Alexandria, Egypt, zen@ejust.edu.eg
}

\begin{abstract}
A hybrid quadrature phase shift keying-modified multi-pulse pulse-position modulation scheme is proposed as a new modulation technique to improve the performance of conventional quadrature phase shift keying (QPSK) modulation in optical fiber communication systems.
\end{abstract}

\section{Introduction}

Multilevel phase modulations represent important modulation category for optical fiber communications. Coherent detection of phase modulated signals increases the receiver sensitivity over traditional intensity modulated direct detected IMDD signals. Toward further enhancement in the performance of multilevel phase modulations, combinations with other modulations are addressed. One direction is to combine phase modulations with pulse position modulations. However, combining phase modulations with pulse position modulations can enhance both receiver sensitivity and bandwidth-utilization efficiency for ordinary multilevel phase modulation schemes. The idea of combing both multilevel phase modulations with pulse position modulations is recently accessed in many publications $[1,2]$.

\section{Proposed System Model and Characteristics}

In this paper, we propose a modified multi-pulse pulse position modulation and modulate its signal pulses by ordinary QPSK modulation. We call the proposed modulation scheme hybrid quadrature phase shift keying-modified multi-pulse pulse position (hybrid QPSK-modified MPPM). This scheme is proposed to improve the performance of ordinary QPSK modulation in optical fiber communications. Comparing with ordinary QPSK, instead of transmitting consecutive low amplitude QPSK symbols, we transmit less number of large amplitude QPSK symbols and exploit the positions of the transmitted symbols to encode additional bits per frame. Precisely, with frames of size

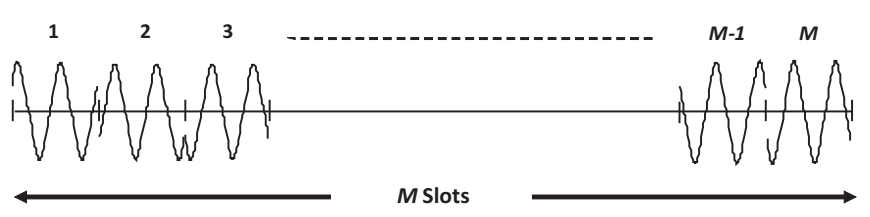

Figure 1. $M$ consecutive symbols in ordinary QPSK scheme.

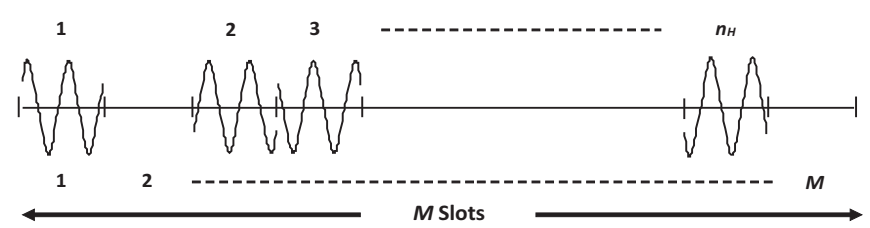

Figure 2. Frame structure in hybrid $n$-QPSK MPPM scheme.

$M$ slots, instead of transmitting $M$ QPSK symbols in ordinary QPSK, $n_{H}$ (less than $M$ ) modulated optical pulses are transmitted in the hybrid frame. The frame structures of ordinary QPSK and proposed hybrid n-QPSK-modified MPPM scheme are explained in Figs. 1, and 2, respectively. The term modified MPPM comes from the ability to increase number of transmitted optical pulses per hybrid frame to values more than $M / 2$.

Obviously, the number of transmitted bits per frame for $M$ consecutive QPSK symbols is $2 M$ bits whereas for hybrid QPSK-modified MPPM, this number is $2 n_{H}+\log _{2}\left(\begin{array}{c}M \\ n_{H}\end{array}\right)$ bits. The $2 n_{H}$ bits are encoded directly in the QPSK symbols and the $\log _{2}\left(\begin{array}{c}M \\ n_{H}\end{array}\right)$ bits are encoded in the positions of the transmitted QPSK symbols. To conserve the same transmission bits per frame for both schemes, there is a minimum value of 

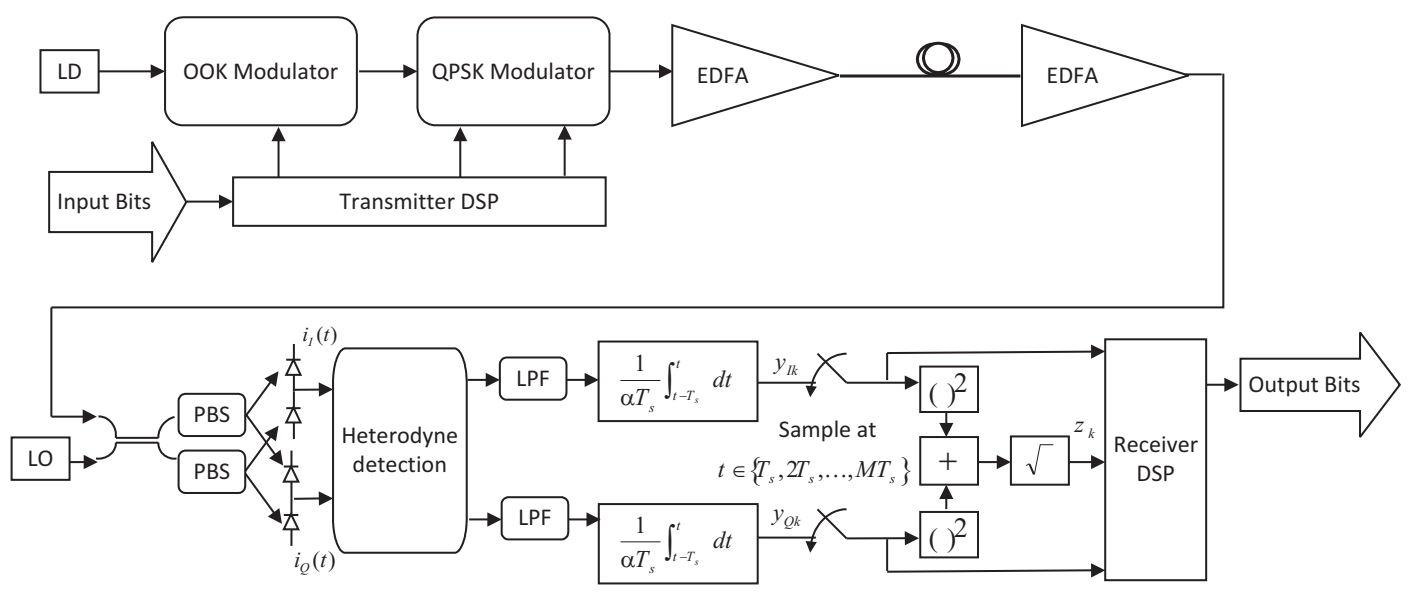

Figure 3. Transmitter and receiver structures for hybrid QPSK-modified MPPM scheme.

$n_{H}$ that could result in near or slightly larger transmission rate for hybrid one. In other words: for any value of $n_{H}$, the maximum frame size $M_{\max }$ that makes the transmission rate for hybrid scheme the same or slightly larger than that of ordinary QPSK scheme is given by

$$
2 n_{H}+\log _{2}\left(\begin{array}{c}
M_{\max } \\
n_{H}
\end{array}\right) \geqslant 2 M_{\max }
$$

Here, we propose to use coherent detection (either homodyne or heterodyne), for demodulating both QPSK and MPPM symbols in the hybrid frame. The transmitter and the receiver structures for hybrid QPSK-modified MPPM are shown in Fig. 3. At the transmitter side, the transmitted data bits are first fed to a digital signal processing (DSP) device, which divides them into several blocks. Each block contains $\log _{2}\left(\begin{array}{c}M \\ n_{H}\end{array}\right)+2 n_{H}$ bits. The first $\log _{2}\left(\begin{array}{c}M \\ n_{H}\end{array}\right)$ bits determine the transmitted MPPM symbol, i.e., the positions of the $n_{H}$ signal pulses within the MPPM frame, while, the remaining $2 n_{H}$ bits are used to modulate the signal pulses with phases of QPSK modulation.

\section{Optimal Decoding Algorithm}

1. Perform coherent detection for the received inand quadrature-phase components.

2. Get the received signal amplitudes for both inand quadrature-phase components in each slot.

3. Determine the energy received for both components in each slot.

4. Get the square root of the sum of components' energies in each slot.

5. Determine $n_{H}$ signal slots with the highest square root values.
6. Decode the QPSK signals of these slots by comparing their integrators outputs to zero.

\section{Performance Evaluation}

Here, we aim at evaluating the bit-error rate (BER) for the proposed hybrid QPSK-modified MPPM. Toward that, the optical fiber is considered as the transmission medium and the system is assumed to be limited by the amplified spontaneous emission (ASE) noise generated from the optical amplifiers [3]. The BER for the hybrid QPSK-modified MPPM scheme is given by

$$
\begin{aligned}
\mathrm{BER}_{\mathrm{H}}= & \frac{\log _{2}\left(\begin{array}{c}
M \\
n_{H}
\end{array}\right)}{2 n_{H}+\log _{2}\left(\begin{array}{c}
M \\
n_{H}
\end{array}\right)} \times \mathrm{BER}_{\mathrm{MPPM}}+\frac{2 n_{H}}{2 n_{H}+\log _{2}\left(\begin{array}{c}
M \\
n_{H}
\end{array}\right)} \\
& \left\{\left(1-\mathrm{SER}_{\mathrm{MPPM}}\right) \times \mathrm{BER}_{\mathrm{QPSK}}+\frac{\mathrm{SER}_{\mathrm{MPPM}}}{\left.n_{H}\left[\begin{array}{c}
M \\
n_{H}
\end{array}\right)-1\right]}\right. \\
& \left.\sum_{i=1}^{\left\lfloor n_{H}, M-n_{H}\right\rfloor} X_{i}\left[\left(n_{H}-i\right) \times \mathrm{BER}_{\mathrm{QPSK}}+\frac{i}{2}\right]\right\} \quad(2)
\end{aligned}
$$

where, $\mathrm{BER}_{\mathrm{QPSK}}$ is the bit-error rate of ordinary QPSK, BER MPPM $_{\text {and }}$ SER $_{\text {MPPM }}$ are the bit-error rate and the symbol-error rate for ordinary MPPM scheme, respectively. For MPPM scheme that transmits $L$ bits per frame, the relation between SER $_{M P P M}$ and $\mathrm{BER}_{\mathrm{MPPM}}$ is given as [4]

$$
\text { BER }_{\text {MPPM }} \leqslant \frac{2^{L-1}}{2^{L}} \times \mathrm{SER}_{\mathrm{MPPM}}
$$

Also, $X_{i}$ represents number of MPPM symbols that differ with $i$ slots from the transmitted symbol. The value of $X_{i}$ could be calculated as 


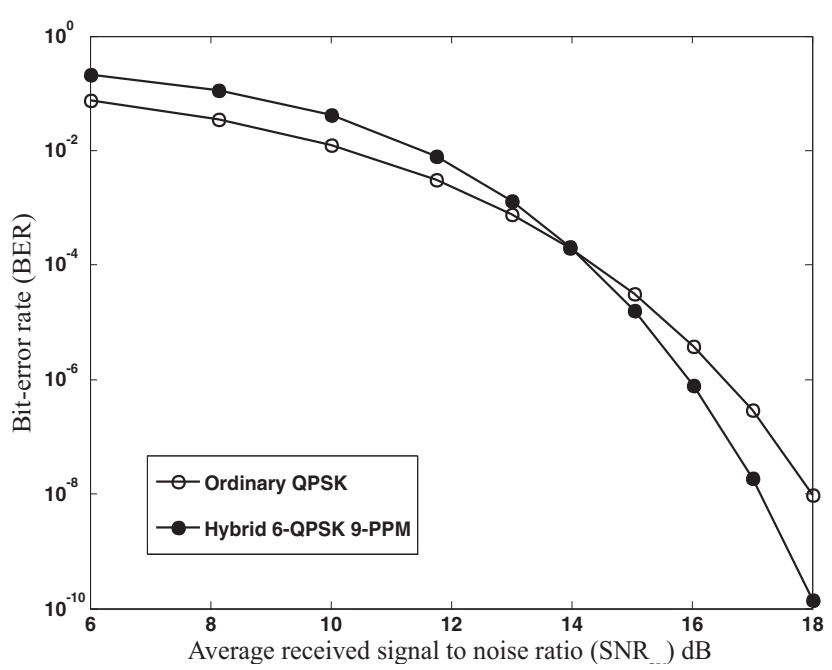

Figure 4. Bit-error rate versus average received signal-to-noise ratio for hybrid 6-QPSK 9-PPM scheme.

$$
X_{i}=\left(\begin{array}{c}
n_{H} \\
i
\end{array}\right) \times\left(\begin{array}{c}
M-n_{H} \\
i
\end{array}\right)
$$

The first term in Eq.(2) accounts for the errors that occur to the group of $\log _{2}\left(\begin{array}{c}M \\ n_{H}\end{array}\right)$ bits which is the same as the bit-error rate of ordinary MPPM. The second term of the equation accounts for bit-error rate of the remaining $2 n_{H}$ bits.

\section{Numerical Results}

In this section, we compare the performance achieved by the proposed hybrid QPSK-modified MPPM with performance achieved by ordinary QPSK under the average power constraint. For fair performance comparison, we assume the usage of same frame size and same transmission bit rate for both schemes. Specifically, the evaluations are performed for two hybrid modulation schemes which are hybrid 6QPSK 9-PPM and hybrid 12-QPSK 22-PPM. For these schemes, the numbers of transmitted bits per hybrid frame are 18.39 bits and 45.36 bits, respectively, which are slightly larger than that of the ordinary QPSK schemes (18 and 44 bits, respectively). Also, in terms of the average received optical power, comparisons are performed under the average power constraint.

The bit-error rates (BERs) versus the average signal-to-noise ratio $\mathrm{SNR}_{a v}$ are plotted in Figs. 4 and 5 for hybrid 6-QPSK 9-PPM and hybrid 12-QPSK 22-PPM schemes, respectively. Cleary, at small values of $\mathrm{SNR}_{a v}$, ordinary QPSK achieves better performance (less BER) than that of hybrid schemes.

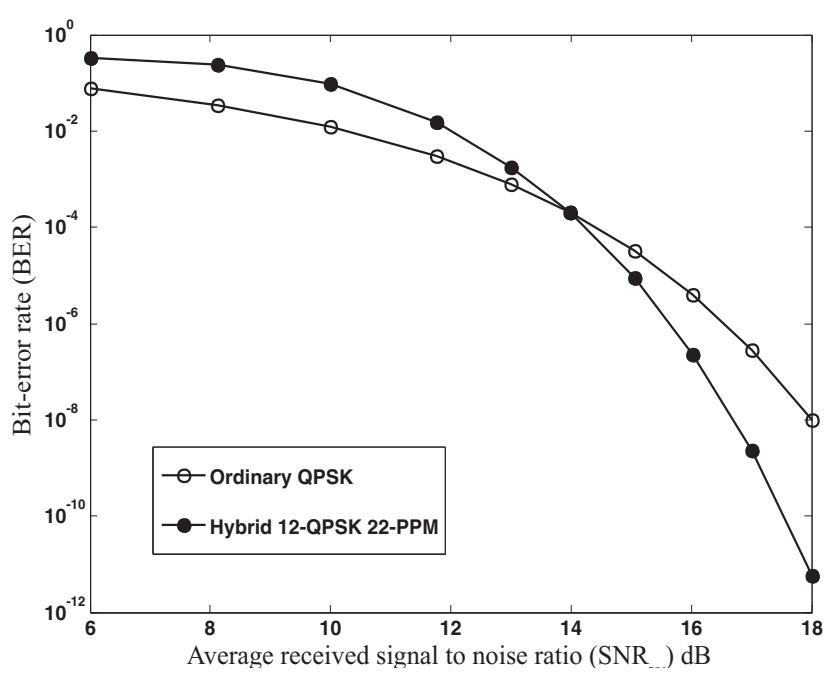

Figure 5. Bit-error rate versus average received signal-to-noise ratio for hybrid 12-QPSK 22-PPM scheme.

While, at moderate and high values of $\mathrm{SNR}_{a v}$, our proposed hybrid schemes outperform the ordinary QPSK scheme. Numerically, as indicated in Fig. 4, at $\mathrm{BER}=10^{-8}$, a reduction of $1 \mathrm{~dB}$ in $\mathrm{SNR}_{a v}$ could be achieved by the hybrid 6-QPSK 9-PPM scheme. The performance gap between two schemes is much increased by raising the level of $\mathrm{SNR}_{a v}$. Moreover, as indicated in Fig. 5, increasing frame size results in better performance for the proposed hybrid scheme.

\section{Conclusions}

A new hybrid modulation, hybrid QPSKmodified MPPM has been proposed for optical communications. The performance measure of the proposed scheme in terms of an upper bound for BER was obtained. Intensive numerical evaluations were performed to compare the performance achieved by the proposed hybrid scheme with the performance of ordinary QPSK scheme. The evaluations were carried out under the average power constraint at nearly the same transmission rates for hybrid and ordinary schemes. At Moderate and high levels of signal-tonoise ratio, the proposed scheme achieves much less bit error rate levels than that of traditional optical QPSK modulation.

\section{References}

[1] M. Karlsson et al., Eur. Conf. Opt. Commun. Tu.6.B.6. (2011).

[2] X. Liu et al., Eur. Conf. Opt. Commun. Tu.3.B.4 (2011).

[3] F. M. K.-P. Ho, Phase-modulated optical communication systems, Publishing Springer (2005).

[4] N. Aoki et al., IEICE Trans. Commun. E79-B, 52-56 (1996). 